\title{
Health disparities and stroke: the influence of insurance status on the prevalence of patient safety indicators and hospital-acquired conditions
}

\author{
Kyle M. Fargen, MD, MPH, Dan Neal, MS, Spiros L. Blackburn, MD, Brian L. Hoh, MD, \\ and Maryam Rahman, MD, MS \\ Department of Neurosurgery, University of Florida, Gainesville, Florida
}

OBJECT The Agency for Healthcare Research and Quality patient safety indicators (PSIs) and the Centers for Medicare and Medicaid Services hospital-acquired conditions (HACs) are publicly reported quality metrics linked directly to reimbursement. The occurrence of PSIs and HACs is associated with increased mortality and hospital costs after stroke. The relationship between insurance status and PSI and HAC rates in hospitalized patients treated for acute ischemic stroke was determined using the Nationwide Inpatient Sample (NIS) database.

METHODS The NIS was queried for all hospitalizations involving acute ischemic stroke between 2002 and 2011. The rate of each PSI and HAC was determined by searching the hospital records for ICD-9 codes. The SAS statistical software package was used to calculate rates and perform multivariable analyses to determine the effects of patient variables on the probability of developing each indicator.

RESULTS The NIS query revealed 1,507,336 separate patient admissions that had information on both primary payer and hospital teaching status. There were 227,676 PSIs (15.1\% of admissions) and 42,841 HACs reported (2.8\%). Patient safety indicators occurred more frequently in Medicaid/self-pay/no-charge patients (19.1\%) and Medicare patients $(15.0 \%)$ than in those with private insurance $(13.6 \% ; p<0.0001)$. In a multivariable analysis, Medicaid, self-pay, or nocharge patients had significantly longer hospital stays, higher mortality, and worse outcomes than those with private insurance $(p<0.0001)$.

CONCLUSIONS Insurance status is an independent predictor of patient safety events after stroke. Private insurance is associated with lower mortality, shorter lengths of stay, and improved clinical outcomes.

http://thejns.org/doi/abs/10.3171/2014.12.JNS14646

KEY WORDS patient safety indicators; hospital-acquired condition; insurance; pay for performance; stroke; vascular disorders

$\mathrm{W}$ ITH the initiation of the Patient Protection and Affordable Care Act, greater attention has been directed toward disparities in health care based on insurance status. The relationship between the lack of health insurance and poorer control of chronic medical conditions is well known. ${ }^{1,2}$ Recently, an increasing number of studies reporting on socioeconomic disparities in ischemic and hemorrhagic stroke have been published in the medical literature. ${ }^{3,6-8,10,13,16-18,20}$ While it has been suggested that private insurance may be a predictor of im- proved outcomes after stroke, this conclusion is not well substantiated.

The Agency for Healthcare Research and Quality (AHRQ) patient safety indicators (PSIs) and the Centers for Medicare and Medicaid Services (CMS) hospital-acquired conditions (HACs) are publically reported quality metrics linked directly to reimbursement for services. The occurrence of PSIs and HACs are common in patients hospitalized for acute ischemic stroke and are associated with increased mortality, longer hospital stays, and increased

ABBREVIATIONS AHRQ = Agency for Healthcare Research and Quality; CMS = Centers for Medicare and Medicaid Services; ENR = estimated national rate; HAC = hospital-acquired condition; NIS = Nationwide Inpatient Sample; PSI = patient safety indicator.

ACCOMPANYING EDITORIAL See pp 868-869. DOI: 10.3171/2014.8.JNS14966.

SUBMITTED March 22, 2014. ACCEPTED December 9, 2014.

INCLUDE WHEN CITING Published online February 6, 2015; DOI: 10.3171/2014.12.JNS14646.

DISCLOSURE The authors report no conflict of interest concerning the materials or methods used in this study or the findings specified in this paper. Dr. Hoh serves on the Steering Committee for an FDA trial evaluating a product of Edge Therapeutics. 
hospital costs. ${ }^{15}$ The relationship between insurance status and these events is currently unknown. To better understand the relationship between PSIs and HACs, and insurance status in stroke patients, hospitalizations for acute ischemic stroke were analyzed using the Nationwide Inpatient Sample (NIS) database.

\section{Methods}

Access to the NIS database was obtained from the AHRQ Healthcare Cost and Utilization Project. For each sampled hospital, all inpatient admissions for the year are contained in the NIS. The NIS includes data for approximately 8 million hospital admissions each year, or approximately one-fifth of all inpatient admissions to US nonfederal hospitals. For more information regarding the NIS database, see http://www.hcup-us.ahrq.gov/nisoverview.jsp. Institutional review board approval was obtained prior to accessing the NIS database for this study.

The NIS was queried for all hospitalizations involving acute ischemic stroke between 2002 and 2011. These hospitalizations were identified in the database using ICD-9 diagnosis codes for stroke (433.01, 433.22, 433.21, 433.31, 433.81, 433.91, 434.01, 434.11, 434.91, 437.1) and cerebral ischemia (435.0, 435.1, 435.2, 435.3, 435.8, 435.9). Insurance status (primary payer) was categorized as Medicaid, Medicare, private insurance, no charge, and self-pay based on NIS state-dependent designations. Admissions with an insurance status listed as "other" were excluded from the analysis $(30,517$ or $2.0 \%$ of hospitalizations). Next, the NIS was mined for the prevalence of AHRQ and HAC ICD-9 diagnosis and procedure codes during each hospitalization. An SAS statistical software package (version 9.3, SAS Institute Inc.) was used to calculate the means, standard deviations, and frequencies for all patient and hospital characteristics and to estimate all PSI and HAC incidence rates. Nationwide Inpatient Sample sampling weights were applied to estimate national rates and to construct confidence intervals for those rates.

Generalized estimating equations were used to assess possible associations between patient and hospital characteristics and AHRQ and CMS indicators. The patient's age, sex, race, comorbidity score, and primary payer status (Medicare, Medicaid, self-pay or no charge, private insurance), and hospital type (teaching vs nonteaching), bed size (small, medium, large), and region (South, West, Northeast, Midwest) were included as covariates. The comorbidity score was calculated by summing the number of Elixhauser comorbidities ${ }^{5}$ recorded in the NIS database for each hospitalization. To account for the clustering of observations on hospitals, hospital was treated as a repeated factor, and an exchangeable working correlation was assumed. Assessed outcomes were any indicators with an incidence rate $>0.1 \%$, as well as mean length of stay, good outcome, and mortality. Finally, mean length of stay, good outcome, and mortality were compared among insurance types.

Previously published definitions ${ }^{11}$ of good versus poor clinical outcome based on the NIS discharge disposition were used. Clinical outcome was defined as "good" if the patient was "discharged to home or self-care," "discharged to short-term hospital for inpatient care," "discharged to home under care of an organized home health service organization," "left against medical advice," "discharged to home intravenous provider," "discharged to another institution for outpatient services," "discharged to same institution for outpatient services," or "discharged alive, destination unknown." Clinical outcome was defined as "poor" if the patient was "discharged to skilled nursing facility," "discharged to intermediate care facility," "discharged to hospice," "discharged to hospital-based Medicare-approved swing bed," "discharged to inpatient rehabilitation facility," "discharged to long-term care hospital," or "discharged to nursing facility certified by Medicaid, but not certified by Medicare." Finally, deceased patients were identified by such terms as "expired," "expired at home," "expired in a medical facility," or "expired-place unknown." Statistical significance was determined by a $\mathrm{p}$ value less than 0.05 .

\section{Results}

The NIS query resulted in 1,514,565 separate patient admissions containing at least one ICD-9 diagnosis code for stroke or cerebral ischemia and a designated insurance status of Medicare, Medicaid, private insurance, self-pay, or no charge. Of these hospitalizations, 1,507,336 had information on both primary payer and hospital teaching status. Medicare patients were significantly older (mean age 77.6 years) than Medicaid patients (mean 54.1 years), patients with private insurance (mean 58.8 years), self-pay/ no-charge patients (mean 54.1 years), and Medicaid/selfpay/no-charge patients (mean 54.1 years; $\mathrm{p}<0.0001$ for all comparisons). Private-insurance patients were also significantly older than Medicaid patients and Medicaid/selfpay/no-charge patients ( $\mathrm{p}<0.0001$ for both comparisons). Table 1 demonstrates the number of hospitalizations based on insurance status. Teaching hospitals treated more Medicaid and fewer privately insured patients than nonteaching hospitals $(\mathrm{p}<0.0001)$.

There were a total of 227,676 PSIs among 1,507,336 hospitalizations, amounting to a PSI prevalence of $15.1 \%$ during admissions for stroke or cerebral ischemia (estimated national rate [ENR] 15.1\%, 95\% CI 14.85\%-15.44\%). There were 42,841 HACs reported, resulting in an ENR of $2.8 \%(95 \%$ CI $2.79 \%-2.92 \%)$.

\section{Total Prevalence of PSIs and HACs, by Insurance Status}

Total PSI and HAC results by insurance status are

TABLE 1. Number of stroke hospitalizations based on primary payer status, 2002-2011

\begin{tabular}{lcrc}
\hline \multirow{2}{*}{$\begin{array}{c}\text { Primary Payerl } \\
\text { Insurance } \\
\text { Status }\end{array}$} & $\begin{array}{c}\text { Teaching } \\
\text { Hospital }\end{array}$ & $\begin{array}{c}\text { Nonteaching } \\
\text { Hospital }\end{array}$ & Total \\
\cline { 2 - 4 } Medicaid & $48,853(52.0)$ & $45,113(48.0)$ & $93,966(6.2)$ \\
\hline Medicare & $400,526(38.1)$ & $651,226(61.9)$ & $1,051,752(69.8)$ \\
\hline Private & $137,790(45.9)$ & $162,627(54.1)$ & $300,417(19.9)$ \\
\hline SP or NC & $29,573(48.3)$ & $31,628(51.7)$ & $61,201(4.1)$ \\
\hline Total & $616,742(40.9)$ & $890,594(59.1)$ & $1,507,336$ \\
\hline
\end{tabular}

$\mathrm{NC}=$ no charge $; \mathrm{SP}=$ self-pay. 
TABLE 2. Total prevalence and ENRs of PSIs and HACs by insurance status

\begin{tabular}{lcccc}
\hline Insurance Status & Total PSIs & ENR $(95 \% \mathrm{CI})$ & Total HACs & ENR $(95 \% \mathrm{CI})$ \\
\hline Private insurance & 40,816 & $13.6 \%(13.2-14.1)$ & 6766 & $2.3 \%(2.2-2.4)$ \\
\hline Medicare & 157,427 & $15.0 \%(14.7-15.3)$ & 31,510 & $3.0 \%(3.0-3.1)$ \\
\hline Medicaid & 21,370 & $22.8 \%(22.1-23.6)$ & 3076 & $3.3 \%(3.1-3.5)$ \\
\hline SP/NC & 8063 & $13.3 \%(12.6-13.9)$ & 1489 & $2.4 \%(2.2-2.7)$ \\
\hline Medicaid \& SP/NC & 29,433 & $19.1 \%(18.5-19.7)$ & 4565 & $3.0 \%(2.8-3.1)$ \\
\hline Total & 227,676 & $15.1 \%(14.9-15.4)$ & 42,841 & $2.8 \%(2.8-2.9)$ \\
\hline
\end{tabular}

depicted in Table 2. At least one PSI occurred in 15,014 Medicaid patients (ENR 16.0\%, 95\% CI 15.5\%-16.5\%), 121,442 Medicare patients (ENR 11.6\%, 95\% CI 11.4\%$11.7 \%$ ), 30,408 privately insured patients (ENR 10.2\%, 95\% CI 9.9\%-10.5\%), and 6127 patients with a primary payer designation of self-pay or no charge (ENR 10.1\%, 95\% CI 9.6\%-10.5\%). At least one HAC occurred in 3076 Medicaid patients (ENR 3.3\%, 95\% CI 3.1\%-3.5\%), 30,336 Medicare patients (ENR 2.9\%, 95\% CI 2.8\%$3.0 \%$ ), 6085 privately insured patients (ENR 2.0\%, 95\%

TABLE 3. Prevalence of individual indicators based on insurance status, 2002-2011

\begin{tabular}{|c|c|c|c|c|c|}
\hline Parameter & Overall & Medicare & Medicaid/SP/NC & Private Insurance & p Value \\
\hline No. of patients & $1,507,336$ & $1,051,752(69.8)$ & $155,167(10.3)$ & $300,417(19.9)$ & \\
\hline \multicolumn{6}{|l|}{ AHRQ indicator (PSI) } \\
\hline Pressure ulcer & $26,002(1.7)$ & $21,175(2.0)$ & $2240(1.4)$ & $2587(0.86)$ & $<0.0001$ \\
\hline latrogenic pneumothorax & $1866(0.12)$ & $1278(0.12)$ & $214(0.14)$ & $374(0.12)$ & 0.228 \\
\hline Central venous line infection & $4430(0.29)$ & $2818(0.27)$ & $668(0.43)$ & $944(0.31)$ & $<0.0001$ \\
\hline Postop hip fracture & $6908(0.46)$ & $6164(0.59)$ & $189(0.12)$ & $555(0.18)$ & $<0.0001$ \\
\hline \multicolumn{6}{|l|}{ Postop physiological derangement-diabetes } \\
\hline w/ ketoacidosis & $2785(0.18)$ & $1403(0.13)$ & $722(0.47)$ & $660(0.22)$ & $<0.0001$ \\
\hline w/ hyperosmolarity & $1204(0.08)$ & $696(0.07)$ & $274(0.18)$ & $234(0.08)$ & $<0.0001$ \\
\hline w/ other coma & $540(0.04)$ & $330(0.03)$ & $107(0.07)$ & $103(0.03)$ & $<0.0001$ \\
\hline Postop hemorrhage & $4222(0.28)$ & $2927(0.28)$ & $386(0.25)$ & $909(0.30)$ & 0.004 \\
\hline Postop respiratory failure & $102,224(6.8)$ & $66,162(6.3)$ & $15,319(9.9)$ & $20,743(6.9)$ & $<0.0001$ \\
\hline Deep vein thrombosis & $17,530(1.2)$ & $11,694(1.1)$ & $2020(1.3)$ & $3816(1.3)$ & $<0.0001$ \\
\hline Pulmonary embolism & $8230(0.55)$ & $5364(0.51)$ & $958(0.62)$ & $1908(0.64)$ & $<0.0001$ \\
\hline Sepsis & $50,013(3.3)$ & 36,266 (3.5) & $6172(4.0)$ & $7575(2.5)$ & $<0.0001$ \\
\hline Accidental puncture or laceration & $1633(0.11)$ & $1088(0.10)$ & $154(0.10)$ & $391(0.13)$ & 0.0002 \\
\hline \multicolumn{6}{|l|}{ CMS indicators (HAC) } \\
\hline Foreign object retained after surgery & $53(0)$ & $40(0)$ & * & * & 0.265 \\
\hline $\begin{array}{l}\text { Pressure ulcer Stages III \& IV (yrs 2008- } \\
2011 \text { only, } n=237,776 \text { ) }\end{array}$ & $2380(0.37)$ & $1891(0.43)$ & $243(0.32)$ & $246(0.19)$ & $<0.0001$ \\
\hline \multicolumn{6}{|l|}{ Falls \& trauma } \\
\hline Fracture & $24,710(1.6)$ & $19,701(1.9)$ & $1721(1.1)$ & $3288(1.1)$ & $<0.0001$ \\
\hline Dislocation & $877(0.06)$ & $612(0.06)$ & $103(0.07)$ & $162(0.05)$ & 0.255 \\
\hline Intracranial injury & $6860(0.46)$ & $4539(0.43)$ & $880(0.57)$ & $1441(0.48)$ & $<0.0001$ \\
\hline Burn & $633(0.04)$ & $415(0.04)$ & $107(0.07)$ & $111(0.04)$ & $<0.0001$ \\
\hline Other injuries & $1413(0.09)$ & $960(0.09)$ & $225(0.15)$ & $228(0.08)$ & 0.0002 \\
\hline Catheter-associated urinary tract infection & $363(0.02)$ & $302(0.03)$ & $25(0.02)$ & $36(0.01)$ & $<0.0001$ \\
\hline $\begin{array}{l}\text { Vascular catheter-associated infection (yrs } \\
2007-2011 \text { only, } n=293,058 \text { ) }\end{array}$ & $1313(0.17)$ & $779(0.14)$ & $234(0.26)$ & $300(0.19)$ & $<0.0001$ \\
\hline \multicolumn{6}{|l|}{ Manifestations of poor glycemic control } \\
\hline Diabetic ketoacidosis & $2785(0.18)$ & $1403(0.13)$ & $722(0.47)$ & $660(0.22)$ & $<0.0001$ \\
\hline Nonketotic hyperosmolar coma & $1204(0.08)$ & $696(0.07)$ & $274(0.18)$ & $234(0.08)$ & $<0.0001$ \\
\hline Deep vein thrombosis & $143(0.01)$ & $116(0.01)$ & * & $23(0.01)$ & 0.003 \\
\hline
\end{tabular}

* Denotes very low number. 
CI 2.0\%-2.1\%), and 1355 self-pay or no-charge patients (ENR 2.2\%, 95\% CI 2.0\%-2.4\%). After combining Medicaid with self-pay or no charge, at least one PSI occurred in 21,141 patients (ENR $13.7 \%, 95 \%$ CI $13.3 \%-14.1 \%$ ) and at least one HAC occurred in 4128 patients (ENR 2.7\%, $95 \%$ CI $2.5 \%-2.8 \%$ ).

In pairwise comparisons of total PSIs between insurance types after controlling for age, sex, comorbidities, and hospital teaching status, bed size, and region, PSIs occurred more frequently in Medicaid patients than in all other groups ( $p<0.0001$ for each pairwise analysis). $\mathrm{Pa}-$ tient safety indicators occurred more frequently in Medicare patients than in privately insured $(\mathrm{p}<0.0001)$ and self-pay/no-charge patients $(\mathrm{p}<0.0001)$. Finally, PSIs occurred less frequently in privately insured patients than in Medicaid/self-pay/no-charge patients ( $p<0.0001)$.

In pairwise comparisons of total HACs between insurance types, HACs occurred more frequently in Medicaid patients than in all other groups $(\mathrm{p}<0.0001$ for each comparison). Similarly, HACs occurred more frequently in Medicare patients than in both privately insured $(\mathrm{p}$ $<0.0001)$ and self-pay/no-charge patients ( $\mathrm{p}<0.0001)$. Hospital-acquired conditions occurred more frequently in Medicaid/self-pay/no-charge patients than in privately insured patients $(\mathrm{p}<0.0001)$.

\section{Prevalence of Individual PSIs and HACs, by Insurance Status}

Table 3 demonstrates the total prevalence of individual PSIs and HACs by insurance status. Indicators with very low rates across all payer types have been excluded from the table. Note that exact totals for indicators with fewer than 10 occurrences are not reported but marked with an asterisk, as required by NIS reporting standards. Overall, the most common PSIs and HACs were postoperative respiratory failure (6.8\% of patients), sepsis (3.3\%), AHRQ pressure ulcer (1.7\%), and falls and trauma-fracture $(1.6 \%)$.

Table 4 demonstrates the prevalence of individual PSIs and HACs by insurance status on multivariable analysis after controlling for age, race, sex, comorbidity score, teaching hospital status, hospital bed size, and hospital region. As Medicare patients are significantly older than patients using other insurance types, Medicare has been excluded from the analysis to prevent significant confounding. On multivariable analysis, AHRQ pressure ulcer, postoperative physiological derangement-diabetic ketoacidosis, postoperative respiratory failure, sepsis, pressure ulcer Stages III and IV, and manifestations of poor glycemic control-diabetic ketoacidosis were significantly more frequent in Medicaid/self-pay/no-charge patients than in privately insured patients. Conversely, postoperative hemorrhage, deep vein thrombosis, pulmonary embolism, and accidental puncture or laceration were significantly more common in privately insured patients than in patients with Medicaid/self-pay/no charge.

\section{Effect of Insurance Status on Outcome Measures}

Table 5 shows the results of multivariable analyses evaluating outcome measures based on insurance status.
TABLE 4. Prevalence of individual PSIs and HACs, Medicaid/SP/ NC versus private insurance

\begin{tabular}{lcc}
\hline \multicolumn{1}{c}{ PSI or HAC } & OR $(95 \%$ Cl) & p Value \\
\hline AHRQ pressure ulcer & $1.6(1.4-1.7)$ & $<0.0001$ \\
\hline latrogenic pneumothorax & $1.0(0.845-1.22)$ & 0.78 \\
\hline Central venous line infection & $0.89(0.785-1.00)$ & 0.06 \\
\hline Postop hip fracture & $1.0(0.839-1.29)$ & 0.73 \\
\hline $\begin{array}{l}\text { Postop physiological derange- } \\
\text { ment-diabetes w/ ketoacidosis }\end{array}$ & $1.4(1.3-1.6)$ & $<0.0001$ \\
\hline Postop hemorrhage & $0.8(0.7-0.9)$ & 0.002 \\
\hline Postop respiratory failure & $1.2(1.1-1.2)$ & $<0.0001$ \\
\hline Deep vein thrombosis & $0.9(0.8-1.0)$ & 0.01 \\
\hline Pulmonary embolism & $0.8(0.7-0.9)$ & $<0.0001$ \\
\hline Sepsis & $1.3(1.2-1.4)$ & $<0.0001$ \\
\hline Accidental puncture or laceration & $0.6(0.5-0.8)$ & $<0.0001$ \\
\hline $\begin{array}{l}\text { Pressure ulcer Stages III \& IV } \\
\text { (yrs 2008-2011 only) }\end{array}$ & $1.6(1.3-1.9)$ & $<0.0001$ \\
\hline $\begin{array}{l}\text { Falls \& trauma-fracture } \\
\text { Falls \& trauma-intracranial injury }\end{array}$ & $0.99(0.877-1.11)$ & 0.84 \\
\hline $\begin{array}{l}\text { Vascular catheter-associated } \\
\text { infection (yrs 2007-2011 only) }\end{array}$ & $0.89(0.738-1.07)$ & 0.20 \\
\hline $\begin{array}{l}\text { Manifestations of poor glycemic } \\
\text { control-diabetic ketoacidosis }\end{array}$ & $1.4(1.3-1.6)$ & $<0.0001$ \\
\hline
\end{tabular}

These measures include length of stay, in-hospital mortality, and poor clinical outcome based on discharge disposition. All outcome measures ( $p<0.0001$ for each) were significantly higher for Medicaid patients than for patients with private insurance.

\section{Discussion}

This study represents the largest and most comprehensive statistical analysis of the effect of insurance status on stroke outcomes in the US. This review of the NIS database of patients admitted with a diagnosis of stroke or cerebral ischemia demonstrates several important phenomena. First, PSIs and HACs are significantly more common in patients with Medicaid than in patients with all other primary payer types, with PSIs occurring in almost 23\% of admissions and HACs occurring in over 3\% of hospitalizations. Second, after controlling for important confounding variables, average length of stay was significantly longer for patients with Medicaid/self-pay/no charge than for those with private insurance by more than 2 days. Furthermore, Medicaid/self-pay/no-charge patients had 1.5 times greater odds of having a poor outcome and 1.2 times the odds of dying during the initial hospitalization than patients with private insurance.

Socioeconomic disparities have been identified in prior studies of ischemic and hemorrhagic stroke. . $^{3,6-8,10,13,16-18,20}$ In a recent retrospective chart review of the New Jersey Acute Stroke Registry of patients presenting with acute ischemic stroke, uninsured patients had higher mortality and longer lengths of stay than insured patients. ${ }^{7}$ Previous reviews of the NIS from $2002^{20}$ and $2005^{9}$ revealed 
TABLE 5. Multivariate analysis comparing outcome measures based on insurance status*

\begin{tabular}{lcccc}
\hline \multicolumn{1}{c}{ Outcome Measure } & Medicaid/SP/NC & Private Insurance & OR (95\% Cl) $\dagger$ & $p$ Value \\
\hline Mean length of stay in days $\neq$ & $7.6 \pm 13.7$ & $5.3 \pm 8.0$ & $1.4(1.3-1.6) \S$ & $<0.0001$ \\
\hline Poor outcome & $16.6 \%$ & $14.6 \%$ & $1.5(1.4-1.5)$ & $<0.0001$ \\
\hline In-hospital mortality & $5.1 \%$ & $4.4 \%$ & $1.2(1.2-1.3)$ & $<0.0001$ \\
\hline${ }^{*}$ After controlling for age, race, sex, comorbidity status, teaching hospital status, hospital bed size, and hospital region. & \\
$\dagger$ Comparison for Medicaid/SP/NC versus private insurance. & & \\
$\ddagger$ Mean \pm standard deviation. \\
$\S$ Denotes a 1.4-day-longer length of stay for Medicaid/SP/NC than for private insurance.
\end{tabular}

a higher level of neurological impairment, higher mortality, and longer lengths of stay for uninsured patients. An uninsured status has also been linked to higher mortality and poorer functional status at discharge after intracranial hemorrhage compared with private insurance. ${ }^{10}$ Finally, it has been suggested that hospital reimbursement may be an important determinant of patient mortality after stroke, with increased mortality seen after reimbursement cuts. ${ }^{22}$

The reasons for poorer outcomes in patients without private insurance are varied. Studies have demonstrated that insured patients have greater awareness of stroke risk factors. ${ }^{4}$ Primary prevention is probably worse in the uninsured, and patients without insurance may wait longer to seek treatment than those with insurance. Uninsured patients with carotid atherosclerosis have more advanced disease at the time of treatment than their insured counterparts. ${ }^{8}$ A prospective study of over 15,000 patients in the Atherosclerosis Risk in Communities Study revealed uninsured patients are also more likely to suffer strokes and be unaware of stroke risk factors and less likely than those with insurance to undergo routine check-ups. ${ }^{6}$ Uninsured patients often have reduced access to acute care and may be less likely to receive emergency therapies. ${ }^{3,19}$

Insured patients are also more likely to receive inpatient rehabilitation following stroke than the uninsured. ${ }^{14,21}$ In fact, longer lengths of stay can often reflect difficulties in rehabilitation placement for those without insurance. Furthermore, uninsured patients are more likely to be unable to afford secondary stroke prevention medications once discharged. ${ }^{12}$ Most of the preceding reasons for poorer outcomes are manifested outside of the acute hospital setting, and most physicians would agree that the care provided for each and every patient with stroke is the same regardless of socioeconomic or insurance status. However, most concerning about the present study is the statistically higher rates of patient safety events for those without private insurance while in the hospital, as well as the higher in-hospital mortality, longer lengths of stay, and worse outcomes based on discharge disposition, even after controlling for important variables such as comorbidities and race. This finding implies that the quality of care provided to stroke patients while in the hospital is, to some degree, dependent on insurance status.

It is important to note that, for the purpose of comparisons, this analysis primarily dichotomizes insurance status into private insurance and Medicaid/self-pay/no charge. Identifying uninsured patients in the NIS is difficult given the highly varied, state-dependent reporting of insurance status, particularly among individuals lack- ing Medicaid, Medicare, or private insurance. Given the previously cited association between private insurance and health outcomes, it was decided to compare patients clearly identified in the NIS as having private insurance against those with suboptimal insurance statuses, consisting of the Medicaid/self-pay/no charge groups. Medicare was excluded from direct comparisons, as patients with this insurance were, on average, roughly $15-20$ years older than patients with private insurance or Medicaid/self-pay/ no charge.

Limitations of this study include its retrospective nature and the inherent potential for selection bias. The NIS database is also subject to coding errors and variability in coding. Our data are limited since the NIS database does not allow distinction between preadmission conditions and hospital-acquired diagnoses. Therefore, some of the reported PSIs and HACs may have been present on admission and mistakenly identified as new events. Neurological assessment scales associated with outcome, such as the Glasgow Coma Scale and the National Institutes of Health Stroke Scale, are also not available in the NIS database; therefore, homogeneity of stroke severity cannot be ensured across populations. Finally, the NIS database does not contain outcome measures such as the modified Rankin Scale score or Glasgow Outcome Scale score, so patient outcomes are determined based on previously published ${ }^{11}$ discharge disposition dichotomization into good versus poor outcomes, which is subject to bias and error and may not truly be representative of functional outcome.

\section{Conclusions}

This study represents the largest and most comprehensive statistical analysis of the effect of insurance status on stroke outcomes in the US. Insurance status is an independent predictor of patient safety events after stroke. The absence of private insurance is associated with higher mortality, longer lengths of stay, and worse clinical outcomes. This study highlights significant health care disparities based on insurance status.

\section{References}

1. Baker DW, Sudano JJ, Albert JM, Borawski EA, Dor A: Lack of health insurance and decline in overall health in late middle age. N Engl J Med 345:1106-1112, 2001

2. Baker DW, Sudano JJ, Durazo-Arvizu R, Feinglass J, Witt WP, Thompson J: Health insurance coverage and the risk of decline in overall health and death among the near elderly, 1992-2002. Med Care 44:277-282, 2006 
3. Brinjikji W, Rabinstein AA, Cloft HJ: Socioeconomic disparities in the utilization of mechanical thrombectomy for acute ischemic stroke. J Stroke Cerebrovasc Dis 23:979-984, 2014

4. Durant RW, Parmar G, Shuaib F, Le A, Brown TM, Roth DL, et al: Awareness and management of chronic disease, insurance status, and health professional shortage areas in the REasons for Geographic And Racial Differences in Stroke (REGARDS): a cross-sectional study. BMC Health Serv Res 12:208, 2012

5. Elixhauser A, Steiner C, Harris DR, Coffey RM: Comorbidity measures for use with administrative data. Med Care 36:8-27, 1998

6. Fowler-Brown A, Corbie-Smith G, Garrett J, Lurie N: Risk of cardiovascular events and death-does insurance matter? J Gen Intern Med 22:502-507, 2007

7. Gezmu T, Gizzi MS, Kirmani JF, Schneider D, Moussavi M: Disparities in acute stroke severity, outcomes, and care relative to health insurance status. J Stroke Cerebrovasc Dis 23:e93-e98, 2014

8. Giacovelli JK, Egorova N, Nowygrod R, Gelijns A, Kent KC, Morrissey NJ: Insurance status predicts access to care and outcomes of vascular disease. J Vasc Surg 48:905-911, 2008

9. Hasan O, Orav EJ, Hicks LS: Insurance status and hospital care for myocardial infarction, stroke, and pneumonia. J Hosp Med 5:452-459, 2010

10. James ML, Grau-Sepulveda MV, Olson DM, Smith EE, Hernandez AF, Peterson ED, et al: Insurance status and outcome after intracerebral hemorrhage: findings from Get With The Guidelines-stroke. J Stroke Cerebrovasc Dis 23:283-292, 2014

11. Lawson MF, Neal DW, Mocco J, Hoh BL: Rationale for treating unruptured intracranial aneurysms: actuarial analysis of natural history risk versus treatment risk for coiling or clipping based on 14,050 patients in the Nationwide Inpatient Sample database. World Neurosurg 79:472-478, 2013

12. Levine DA, Kiefe CI, Howard G, Howard VJ, Williams OD, Allison JJ: Reduced medication access: a marker for vulnerability in US stroke survivors. Stroke 38:1557-1564, 2007

13. Li C, Hedblad B, Rosvall M, Buchwald F, Khan FA, Engström G: Stroke incidence, recurrence, and case-fatality in relation to socioeconomic position: a population-based study of middle-aged Swedish men and women. Stroke 39:21912196,2008

14. Ottenbacher KJ, Graham JE: The state-of-the-science: access to postacute care rehabilitation services. A review. Arch Phys Med Rehabil 88:1513-1521, 2007
15. Rahman M, Neal D, Fargen KM, Hoh BL: Establishing standard performance measures for adult stroke patients: a nationwide inpatient sample database study. World Neurosurg 80:699-708.e2, 2013

16. Rey V, Faouzi M, Huchmand-Zadeh M, Michel P: Stroke initial severity and outcome relative to insurance status in a universal health care system in Switzerland. Eur J Neurol 18:1094-1097, 2011

17. Sandel ME, Wang H, Terdiman J, Hoffman JM, Ciol MA, Sidney S, et al: Disparities in stroke rehabilitation: results of a study in an integrated health system in northern California. PM R 1:29-40, 2009

18. Schieb LJ, Mobley LR, George M, Casper M: Tracking stroke hospitalization clusters over time and associations with county-level socioeconomic and healthcare characteristics. Stroke 44:146-152, 2013

19. Schumacher HC, Bateman BT, Boden-Albala B, Berman MF, Mohr JP, Sacco RL, et al: Use of thrombolysis in acute ischemic stroke: analysis of the Nationwide Inpatient Sample 1999 to 2004. Ann Emerg Med 50:99-107, 2007

20. Shen JJ, Washington EL: Disparities in outcomes among patients with stroke associated with insurance status. Stroke 38:1010-1016, 2007

21. Skolarus LE, Meurer WJ, Burke JF, Prvu Bettger J, Lisabeth LD: Effect of insurance status on postacute care among working age stroke survivors. Neurology 78:1590-1595, 2012

22. Tung YC, Chang GM: The effect of cuts in reimbursement on stroke outcome: a nationwide population-based study during the period 1998 to 2007. Stroke 41:504-509, 2010

\section{Author Contributions}

Conception and design: Rahman. Acquisition of data: Rahman. Analysis and interpretation of data: Fargen, Neal, Rahman. Drafting the article: Fargen, Rahman. Critically revising the article: all authors. Reviewed submitted version of manuscript: all authors. Approved the final version of the manuscript on behalf of all authors: Fargen. Statistical analysis: Neal. Study supervision: Hoh, Rahman.

\section{Correspondence}

Kyle M. Fargen, Department of Neurosurgery, University of Florida College of Medicine, Box 100265, Gainesville, FL 32610. email: kyle.fargen@ neurosurgery.ufl.edu. 